\title{
El taladrador de las meliaceas, Hypsipyla grandella (Zellar) (Insecta: Lepidoptera: Pyralidae: Phycitinae) $)^{1}$
}

\section{F. W. Howard y Michael A. Merida ${ }^{2}$ \\ Introducción}

El taladrador de las meliáceas, Hypsipyla grandella (Zeller), taladra los brotes de árboles en la familia de las caobas (Meliaceae), especialmente las caobas (Swietenia spp.) y los cedros (Cedrela spp.). Es una plaga económica importante, y ha sido el objetivo de investigaciones en muchos países tropicales. Es la única especie de Hypsipyla en Florida, donde es una plaga de caoba antillana (Swietenia mahagoni Jacquin), un árbol nativo que es frecuentemente plantado como un árbol de sombra.

Hypsipyla robusta (Moore) y especies cercanas en Madagascar y Àfrica juegan un papel similar como el taladrador de brotes de árboles meliáceos en regiones tropicales del Hemisferio Oriental. Actualmente, se comprende que Hypsipyla robusta es un complejo de especies y su taxonomía está siendo resuelta (Marianne Horak, personal communication). Se han referido a Hypsipyla robusta y talvez a otras especies de este complejo por varios nombres vernáculos en inglés, los cuales se pueden traducir al español como taladrador de toona, polilla del brote de cedro, gusano del brote de cedro, y en algunos países como el taladrador de caoba. En este articulo, el nombre taladrador de las meliáceas se refiere a la especia de las Américas, $H$. grandella. Nueve especies adicionales de Hypsipyla se han descrito. Todas son tropicales (tres en las Américas, y seis en el Hemisferio Oriental). La distribución de cada una de esas es limitada. Ellas no son plagas económicas importantes, y se conoce poco al respecto de su biología (Horak 2001). Revisiones recientes de taladradores Hypsipyla incluyen Newton et al. (1993) y Griffiths (2001). Libros sobre las caobas con discusiones sobre los insectos asociados incluyen Lamb (1966) y Mayhew and Newton (1998). Treinta y seis trabajos fueron publicados en 'Proceedings of a Workshop on Hypsipyla shoot borers' (Floyd and Hauxwell 2001).

\section{Distribución}

La distribución del taladrador de las meliáceas probablemente coincide con la de sus plantas hospederas principales, eso es, las caobas y los cedros, i.e., el sur de la Florida, la mayoría de las islas de las Indias Occidentales, México desde Sinaloa hasta el sur, Centromérica, y América del Sur con la excepción de Chile (Griffiths 2001) (vea a Plantas hospederas).

1. This document is EENY-337, one of a series of Featured Creatures from the Entomology and Nematology Department, Florida Cooperative Extension Service, Institute of Food and Agricultural Sciences, University of Florida. Fecha de Publicación: Diciembre de 2004. This document is also available on Featured Creatures Website at http://creatures.ifas.ufl.edu. Please visit the EDIS Website at http://edis.ifas.ufl.edu. 2. F. W. Howard y Michael A. Merida, Department of Entomology and Nematology, University of Florida, Gainesville, FL.

The Institute of Food and Agricultural Sciences (IFAS) is an Equal Opportunity Institution authorized to provide research, educational information and other services only to individuals and institutions that function with non-discrimination with respect to race, creed, color, religion, age, disability, sex, sexual orientation, marital status, national origin, political opinions or affiliations. U.S. Department of Agriculture, Cooperative Extension Service, University of Florida, IFAS, Florida A. \& M. University Cooperative Extension Program, and Boards of County Commissioners Cooperating. Larry Arrington, Dean 


\section{Descripción}

Segun Ramirez Sanchez (1964), Becker (1976), Solomon (1995):

Adulto. Los adultos de $H$. grandella son de color de marrón a marrón-grisáceo. La envergadura de las alas anteriores es cerca de 23 a $45 \mathrm{~mm}$. Estas son gris-fuscas sombreadas de color ladrillo en la parte posterior de la ala. Las áreas medias a afueras de las alas anteriores aparecen espolvoreadas con escamas y con puntos negros hacia las puntas de las alas. Las venas de las alas son recubiertas con escamas negras. Las alas traseras son blancas a translucidas con margines oscuras. La cabeza, cuerpo, y patas son de un color castaño-grisáceo.

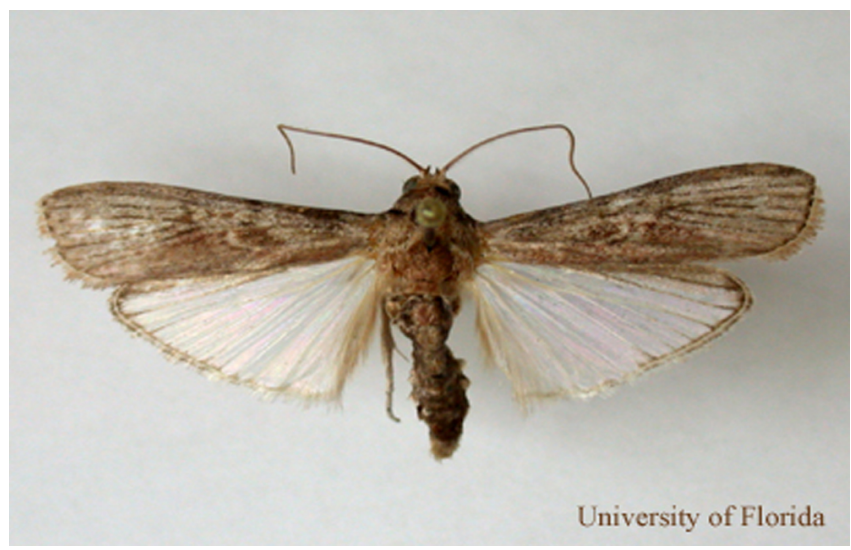

Figure 1. Taladrador de las meliáceas, Hypsipyla grandella (Zeller), polilla preservada en alfiler. Credits: L.J. Buss, University of Florida

Huevo. Los huevos del taladrador de las meliaceas son ovalados, aplanados, y miden aproximadamente $0.9 \mathrm{~mm}$ de largo por $0.5 \mathrm{~mm}$ de ancho. El color de los huevos cambia de blanco a rojizo dentro de 24 horas después de la oviposición.

Larva. El cuerpo de la larva de los instares jóvenes es de un color habano pálido a blanco, volviendo azul en los instares más avanzados. La cápsula de la cabeza es marrón. Las larvas maduras son de aproximadamente $25 \mathrm{~mm}$ de largo.

Pupa. La pupa de H. grandella es marrón-negro en color y envuelto en un cocón de seda.

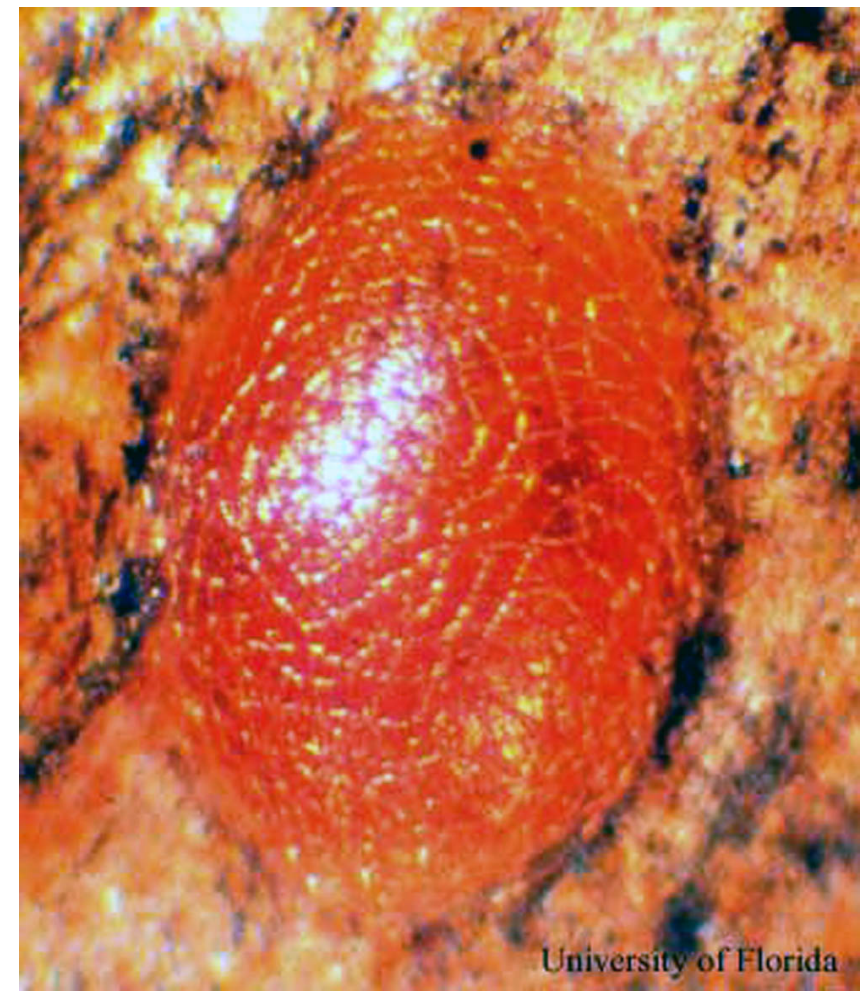

Figure 2. Taladrador de las meliáceas, Hypsipyla grandella (Zeller), huevo. Credits: J.V. DeFilippis, University of Florid

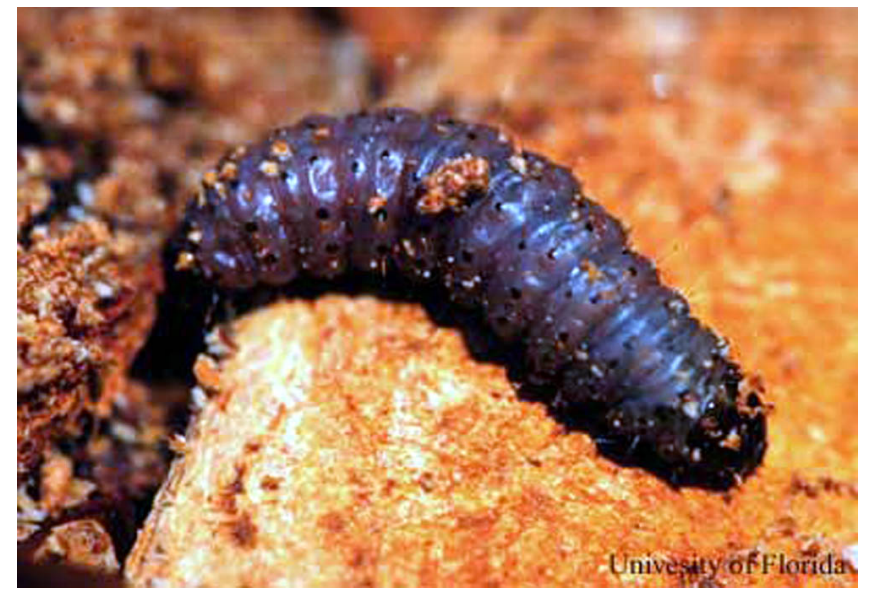

Figure 3. Taladrador de las meliáceas, Hypsipyla grandella (Zeller), larva. Credits: FW Howard, University of Florida

\section{Biología}

Las polillas adultas son nocturnas y viven siete a ocho días (Holsten 1976). Estas depositan los huevos durante las horas tempranas de la mañana sobre brotes nuevos, cicatrices de las hojas caídas, hojuelas (especialmente al lado de una vena en la superficie superior), y frutas. Los huevos generalmente se ponen uno por uno, pero a veces 
están agregados en un grupo de tres o cuatro huevos metidos en axilas de hojas. Una hembra puede poner cerca de 300 huevos, pero típicamente pone no más que unos pocos huevos por cada árbol (Solomon 1995).

En estudios del tiempo de desarrollo del taladrador de las meliáceas alimentándose sobre las hojas frescas de cedro a temperaturas constantes, la duración del estado larval variaba entre $30\left(30^{\circ} \mathrm{C}\right) \mathrm{y}$ 104 días $\left(15^{\circ} \mathrm{C}\right)$ (Taveras et al. 2004). En el campo, la duración total del desarrollo de la larva, prepupa y pupa es completo dentro de uno o dos meses, depende las condiciones ambientales, y se puede extenderse si el insecto pasa por diapause. Las larvas recientemente emergidas muchas veces empiezan a alimentarse de la superficie de la hoja o del brote, después taladran en los brotes o sea en las cápsulas de las semillas. Las larvas las cuales taladran en los brotes producen un túnel de varios centímetros de largo. El tallo hueco se seca y se dobla, y las hojas de este se mueran. Una masa de excremento de color marrón-rojizo entrelazado con hilos de seda del insecto sale de la apertura de entrada. Se puede determinar por la apariencia del excremento expendido si una larva esta activamente taladrando en un brote, pues este es compacto y relativamente pálido en color, volviendo más oscuro y desintegrado después de que la larva ya no está alimentándose (por ejemplo, en el estado de la pupa) (Howard 1991). Al partir un brote infestado, se puede observar la larva o pupa, si uno de estas está adentro.

Los taladradores de las caobas atacan brotes nuevos y raramente atacan los brotes maduros. En el sur de Florida, donde el brote de la caoba antillana toma lugar durante abril-junio (Howard 1991), los taladradores de caobas atacan los brotes desde los principios de primavera hasta el medio de verano, con picos poblacionales en mayo. En los trópicos, el taladrador de las meliáceas está activo todo el año, con la actividad concentrada en la temporada de lluvias, la cual es el período de crecimiento de brotes de las caobas. Se han observado picos poblacionales en la primavera, i.e., en los principios de la temporada de lluvias (Roovers 1971, Bauer 1987).

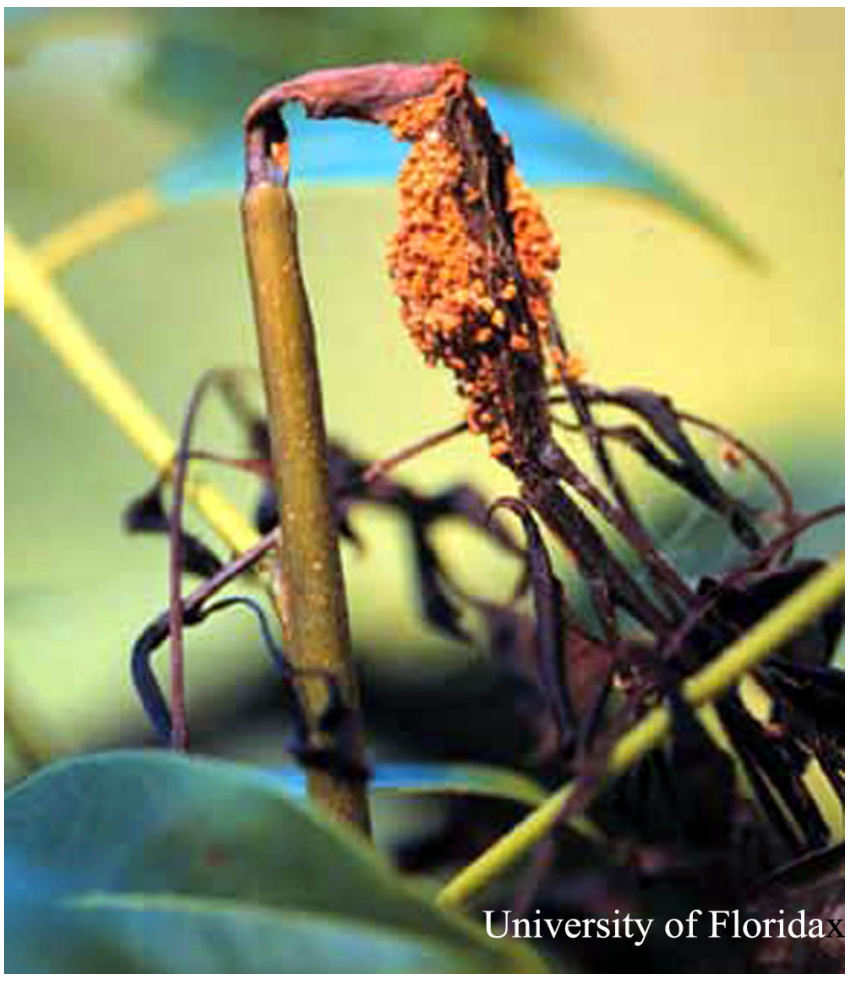

Figure 4. Daño al brote por el taladrador de las meliáceas, Hypsipyla grandella (Zeller). Credits: FW Howard, University of Florida

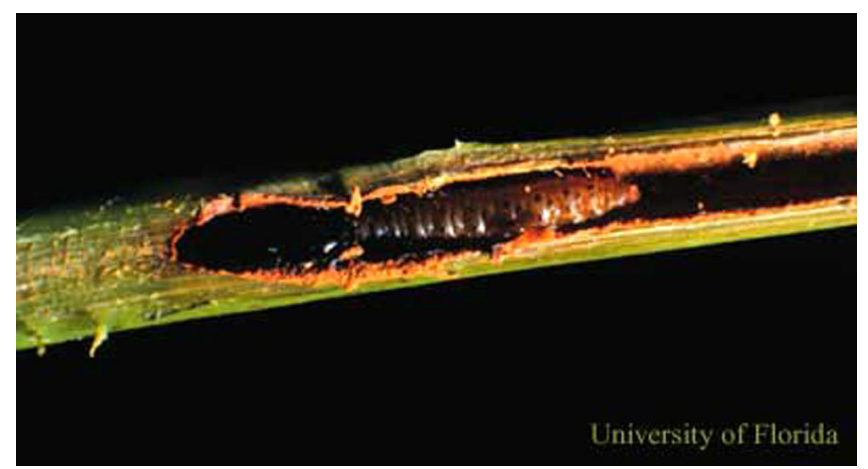

Figure 5. Tallo de caoba antillana partida para revelar larva de taladrador de las meliáceas, Hypsipyla grandella (Zeller). Credits: Jim DeFilippis, University of Florida

El taladrador de las meliáceas también ataca las cápsulas de las semillas de las caobas, los cedros, y otros árboles meliáceos. Según observaciones sobre el ataque de este insecto en caobas antillanas en el sur de la Florida, raramente perforan las válvulas duras de las cápsulas de las semillas, sino penetran ente ellas al abrir la cápsula. En este sitio consumen las 50-80 semillas, luego penetran el corazón de la cápsula donde a veces pasan el estado de la pupa. En la Florida, el ataque del taladrador de las meliáceas sobre cápsulas de semilla de la caoba antillana esta básicamente 


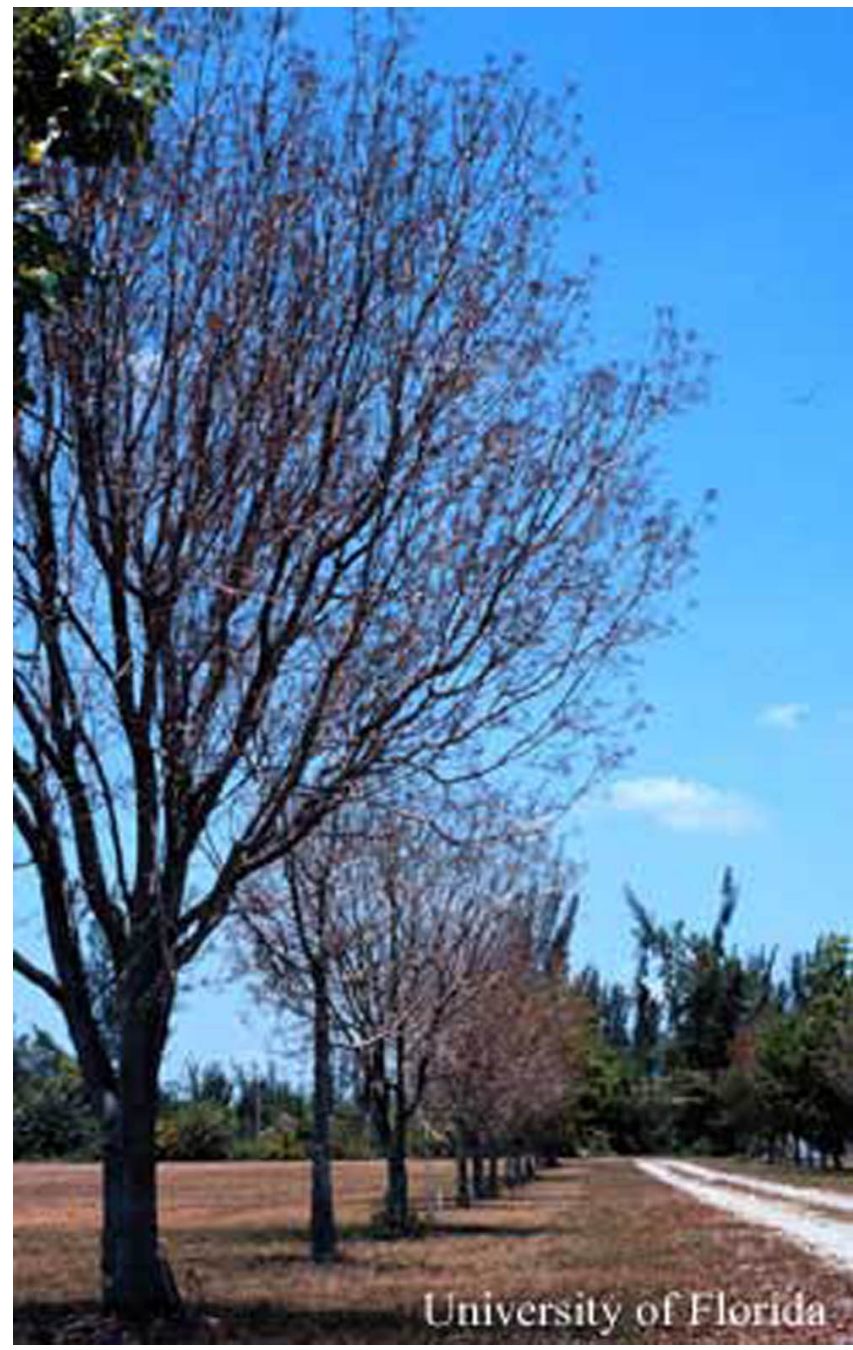

Figure 6. Caoba antillana, Swietenia mahagoni, durante el brote de la primavera. Credits: FW Howard, University of Florida

limitado al período durante la dehiscencia de la cápsula, eso es, en la primavera antes y simultanea con la producción de nuevos brotes.(Howard and Gilblin-Davis 1997). El estadio de la pupa toma lugar adentro del brote hueco o sea en la cápsula de semillas, o ocasionalmente en la capa de hojas muertas o en el suelo debajo los árboles hospederos. El estadio de la pupa dura 8-10 días (Ramírez Sánchez 1964).

\section{Plantas hospederas}

Especies de varios géneros en la familia botánica Meliaceae sirven como plantas hospederas del taladrador de las meliáceas, incluyendo Carapa, Cedrela, Guarea, Khaya, Swietenia, y Trichilia (Entwistle 1967, Becker 1976). La mayoría de las especies son nativas de los trópicos de las

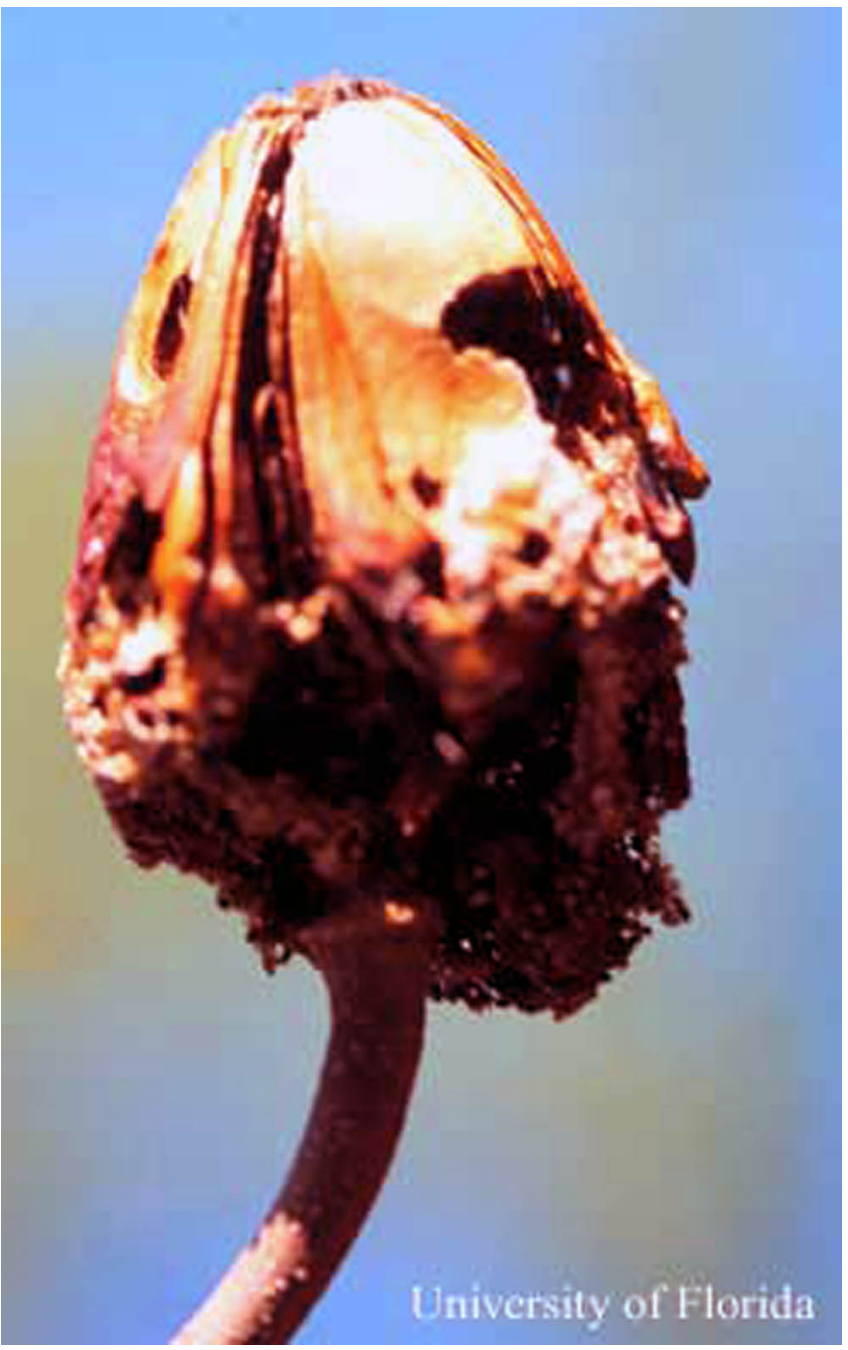

Figure 7. Caoba antillana, Swietenia mahagoni, cápsula de semillas dañado por Taladrador, de las meliááceas, Hypsipyla grandella (Zeller). Credits: FW Howard, University of Florida

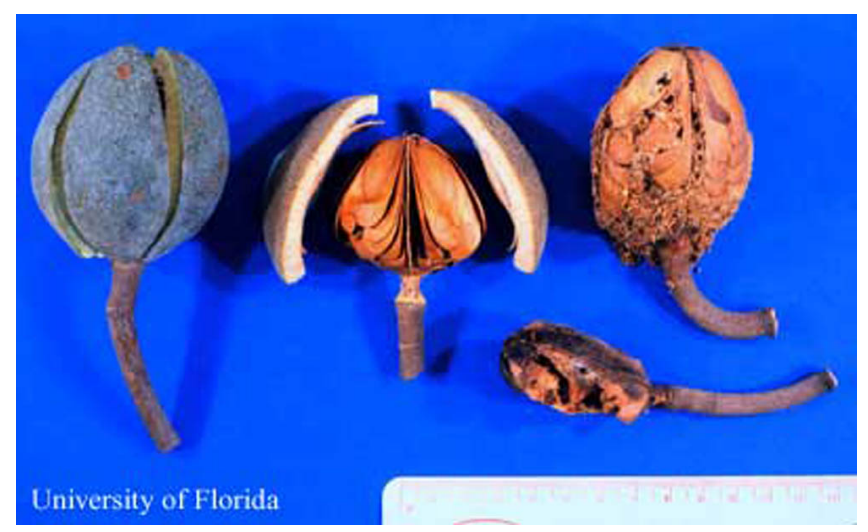

Figure 8. Caoba antillana, Swietenia mahagoni, cápsulas de semillas y partes dañados por el taladrador de las meliáceas, Hypsipyla grandella (Zeller). Izquierda a derecho: Cápsula en estado inicial de dehiscencia, cápsula no atacada, corazón dañado por larvas, cápsula abierta dañada por larvas. Credits: FW Howard, University of Florida 
Américas, pero especies exóticas como Khaya spp. pueden ser atacadas cuando se crecen en América Tropical.

Entre las especies nativas, Cedrela odorata L. tiene la distribución más amplia, ocurriendo en las áreas del continente de las Américas de México norteño a través de la Argentina, y en la mayoría de las islas de las Indias Occidentales (Cintron 1990). El rango de esta especie más o menos coincide con la del taladrador de las meliáceas, con la excepción de que el $C$. odorata no es nativo a la Florida. Hay por lo menos 11 especies de Cedrela (Pennington 1981), y se sabe que el taladrador de las meliáceas ataca por lo menos a algunos de ellos además de atacar al C. odorata (Becker 1976). Los cedros son árboles de crecimiento rápido y son grandes en la madurez. Su madera se utiliza localmente en maneras numerosas a través de las zonas tropicales americanas, y se plantan como árboles de sombra en áreas urbanas de esta región, pero están presentes solamente como árboles de espécimen ocasionales en la Florida.

El nombre vernáculo del taladrador de las meliáceas de la caoba refleja la importancia comercial de unos de sus plantas hospederas, eso es, las caobas. Las caobas verdaderas (Swietenia spp.) son nativas a las zonas tropicales americanas y a algunas áreas afuera de las zonas tropicales con un clima tropical, tales como las Bahamas y Florida meridional. El género incluye las tres especies siguientes (Record and Hess 1943, Lamb 1966, Pennington 1981): La caoba de las Indias Occidentales (S. mahagoni [Jacquin]), que es nativa a la Florida meridional, las Bahamas, y las Antillas Mayores excepto Puerto Rico. Éste es uno de los árboles más populares para uso ornamental o para sombra en áreas urbanas de la Florida meridional, y es un componente de bosques naturales de áreas tales como los Everglades y Los Cayos de la Florida. Es el único árbol meliáceo grande que es común en la Florida.

La caoba hondureña ( $S$. macrophylla King), que es nativa al continente de América Tropical, ocurre en regiones húmedas tropicales desde alrededor de latitud de $22^{\circ} \mathrm{N}$ en el lado atlántico de México a través de Centroamérica y de Sudamérica hasta

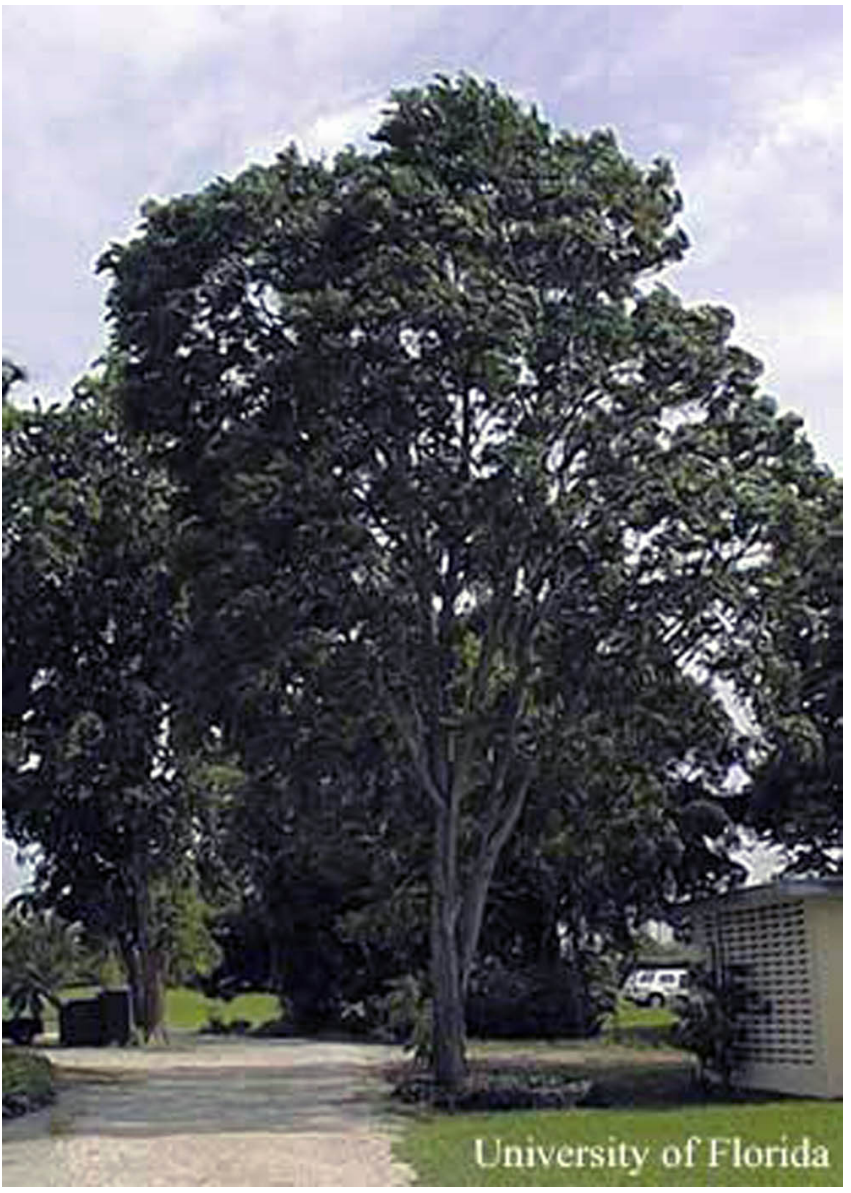

Figure 9. Cedro, Cedrela odorata. Credits: FW Howard, University of Florida

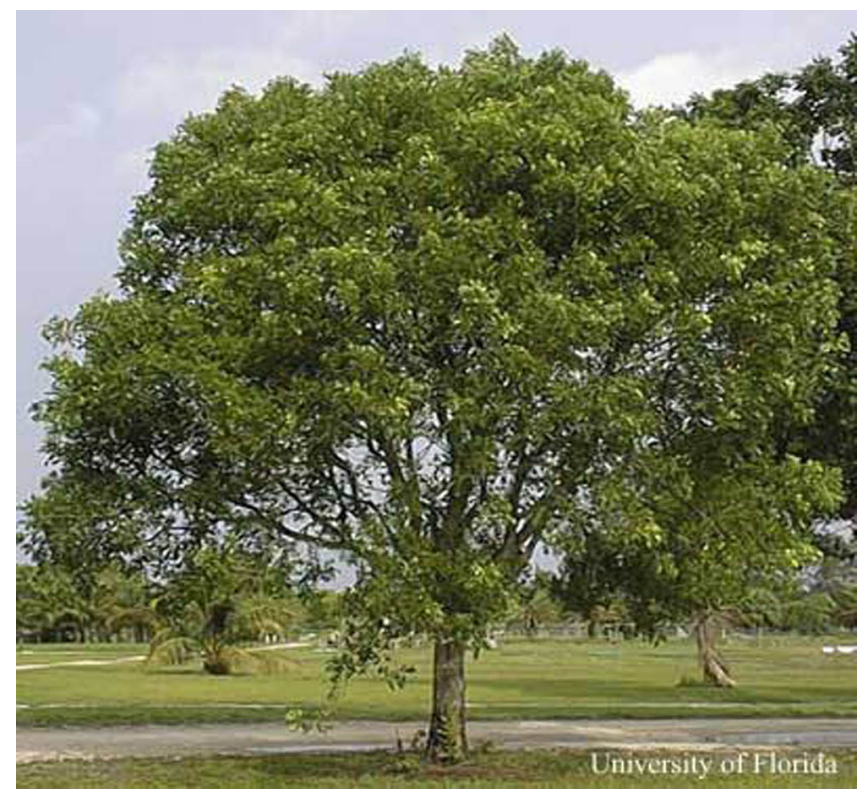

Figure 10. Caoba antillana, Swietenia mahagoni. Credits: FW Howard, University of Florida 


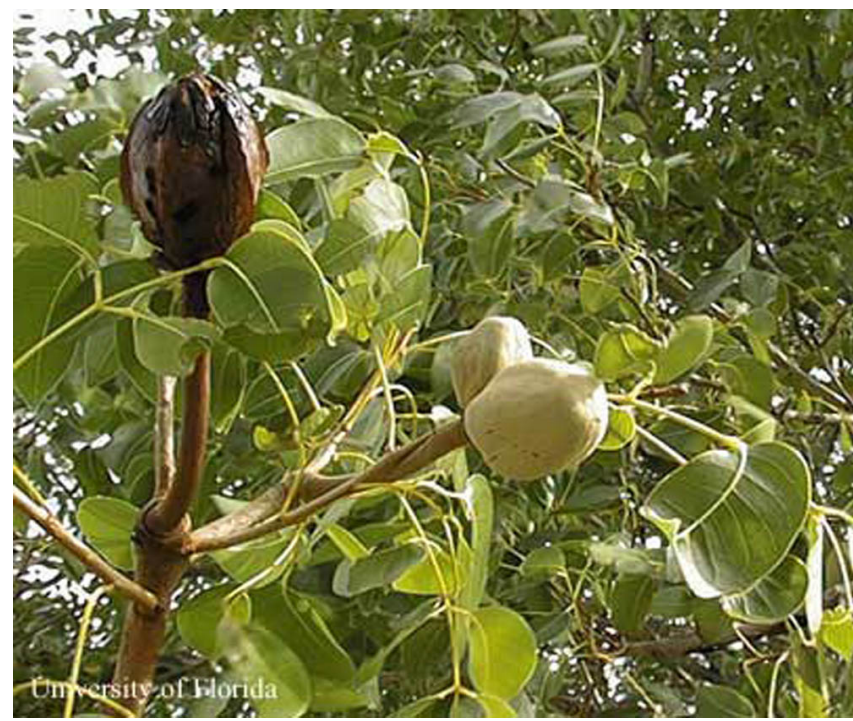

Figure 11. Caoba antillana, Swietenia mahagoni, follaje y cápsulas de semillas. Credits: FW Howard, University of Florida

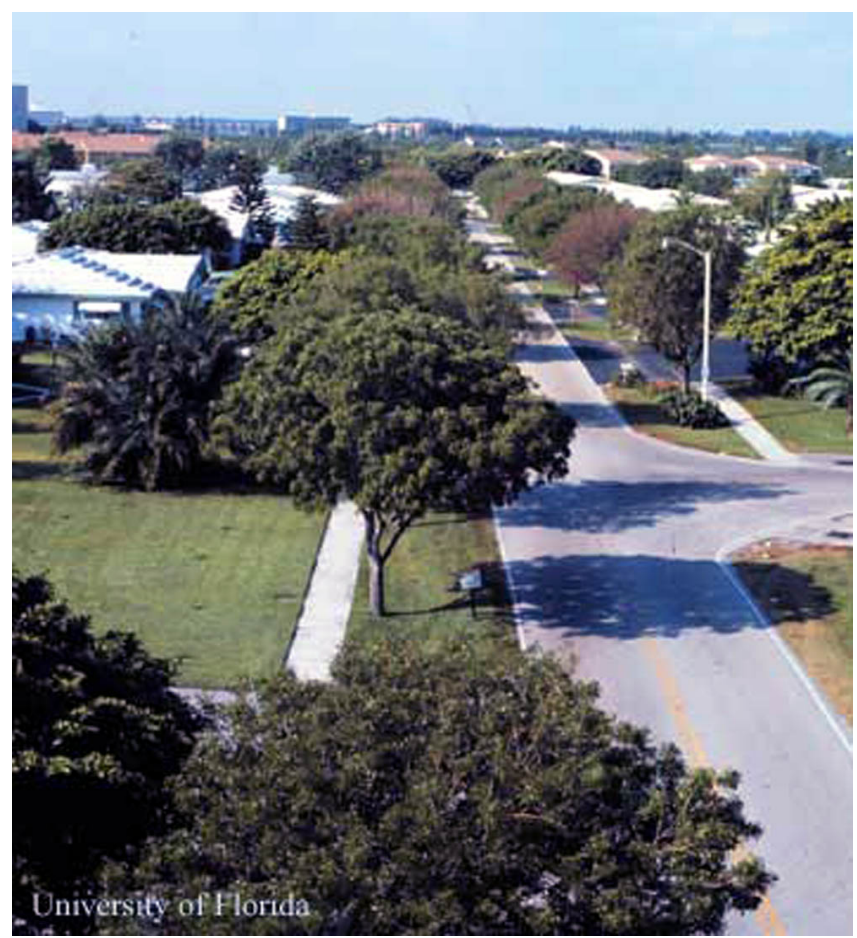

Figure 12. Caoba antillana, Swietenia mahagoni, como árbol de sombra en área urbana de la Florida. Credits: FW Howard, University of Florida

alrededor a la latitud de $22^{\circ} \mathrm{S}$ en Bolivia. Es actualmente la fuente principal de la madera de caoba, pero en la Florida se encuentra ocasionalmente y solamente como un árbol de espécimen.

La caoba del Pacifico (S. humilis Zuccarini) se distribuye al lado de las áreas costeras Pacíficas de

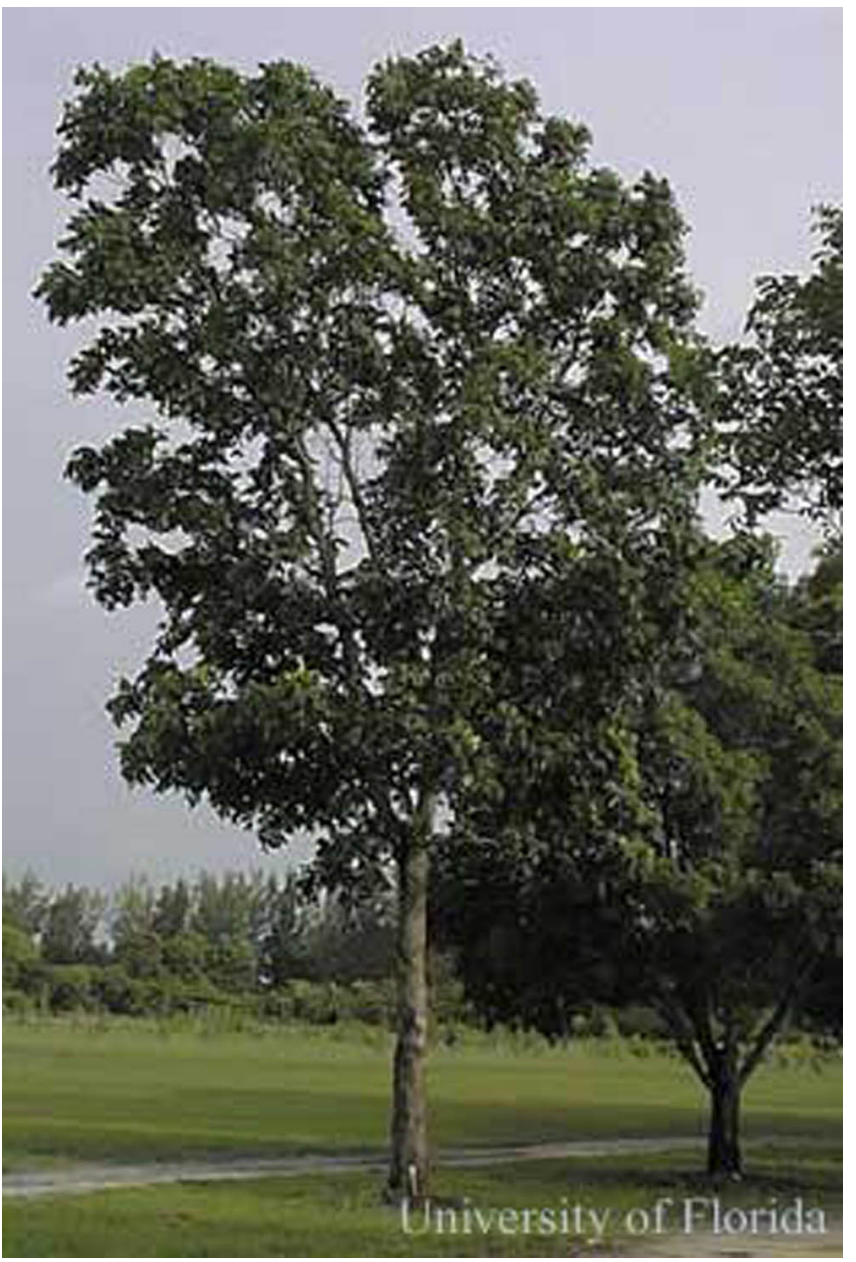

Figure 13. Caoba hondureña, Swietenia macrophylla. Credits: FW Howard, University of Florida

México hasta Costa Rica. En la Florida es un árbol de espécimen raramente visto.

Las caobas verdaderas son quizás los árboles maderables tropicales más importantes del mundo. La madera se usa principalmente como madera de gabinete. Durante la Época de la Colonia en el Caribe la caoba antillana fue explotada extensivamente, y después la caoba hondureña, la cual es presente en el continente de las Américas y es más extensivamente distribuida, se convirtió en la fuente principal de la madera de caoba.

Unas ciertas especies de árboles meliáceas que son nativas a las zonas tropicales del Hemisferio Oriental son atacadas por el taladrador de las meliáceas $(H$. grandella) cuando estas plantas están presentes en las Américas; en sus regiones nativas son generalmente plantas hospederas del contraparte de este insecto en el Hemisferio Oriental, 
eso es, $H$. robusta. Un ejemplo es caoba de Nyasalandia, o caoba africana (Khaya nyasica Baker ex f de Stapf.) (Entwistle 1967, Becker 1976).

Por otra parte, el cedro-rojo (Toona ciliata [ Roemer ] var. australis), y caoba africana (Khaya ivorensis A. Chevallier), ambos árboles meliaceos maderables importantes de las zonas tropicales del Hemisferio Oriental que son atacadas fuertemente por el $H$. robusta al crecer allí, no fueron atacadas por $H$. grandella cuando estaban presentes en Costa Rica (Grijpma 1970).

\section{Daños económicos}

Los árboles de caoba son susceptibles al ataque cuando alcanzan una altura de $0.5 \mathrm{~m}$ (Griffiths 2001), aunque en la Florida se atacan raramente cuando están menos que cerca de $1.0 \mathrm{~m}$ de alto (Howard, inéditos). El daño más severo del insecto a los árboles ocurre cuando una larva taladra en el brote terminal y lo mata. Un ramo lateral crece hacia arriba para substituir al brote terminal perdido, dando por resultado un tallo principal torcido. También, el daño al terminal rompe la dominación apical, dando por resultado una ramificación lateral excesiva.

(Howard and Meerow 1993). Los árboles pequeños que los taladradores atacan fuertemente en varias ocasiones en años sucesivos se deformen extremadamente.

El ataque del taladrador de las meliáceas reduce el grado y así el valor monetario de las caobas jóvenes en viveros donde los cultivan para uso como árboles del paisaje, eso es, su principal uso en la Florida (Howard and Meerow 1993). Porque los ataques últimamente resultan en una reducción en el número y la longitud de troncos rectos y claros, el taladrador del brote da la caoba es una plaga importante de caobas, cedros, y otros árboles maderables meliáceos en las zonas tropicales. Atacan un porcentaje más alto de los árboles donde las caobas se crecen en plantaciones que donde estos árboles crecen entremezclados en bosques naturales, $\mathrm{y}$ este insecto ha sido un impedimento importante al establecimiento de las plantaciones de caoba. Se necesitan urgentemente métodos de cultivar las caobas en plantaciones para disminuir el impacto de cortarlos en bosques naturales (Lamb 1966, Newton et al. 1993, Mayhew and Newton 1998, Floyd and Hauxwell 2001).

Muchos autores han mencionado el daño a las semillas por el taladrador de las meliáceas, e.g., Monte (1933), Tillmanns (1964), Becker (1976), pero éste ha sido generalmente poco importante o en segundo lugar en importancia comparado con el daño a los brotes. Becker (1976) sugirió que la producción de semilla era suficiente para compensar para las pérdidas al taladrador de las meliáceas. Sin embargo, en un estudio en la Florida, el taladrador de las meliáceas atacó hasta $100 \%$ de las cápsulas de semilla por árbol de caoba antillana y consumió entre el $50 \%$ y $96 \%$ de las semillas por cápsula. Durante el mismo período, solamente $14-22 \%$ de brotes nuevos en los árboles fue atacado. El impacto de este insecto en la regeneración se debe investigar más.

\section{Manejo}

La actividad de la investigación para desarrollar métodos de manejo ha sido mucho mayor para el taladrador de las meliáceas como plaga de los árboles maderables más bien que como árboles ornamentales o de sombra (Lamb 1966, Grijpma 1974, Newton et al. 1993, Mayhew and Newton 1998, Floyd and Hauxwell 2001). En cualquier situación, este insecto es supremamente difícil de controlar, sobre todo porque aunque algunos métodos reducen la población de la plaga considerablemente, hasta las poblaciones ligeras pueden causar daño significativo. En hecho, el daño más importante del insecto, destrucción del brote terminal, es el resultado de una sola larva por árbol .

En la Florida, el taladrador de las meliáceas es en gran parte una plaga de la caoba antillana en viveros de macetas y viveros del campo en donde estos árboles nativos se crecen para el uso como árboles de sombra. Las caobas establecidas en el paisaje también son atacadas comúnmente por los taladradores los meliaceas, sobre todo en la primavera, pero el daño no afecta ni el crecimiento perceptiblemente ni la calidad estética de árboles maduros. Solamente se ataca un porcentaje de los brotes crecientes en un árbol, el daño a las ramitas no es visible desde una distancia, y el crecimiento de ramas durante el verano encubre el daño. 
Control químico. Wylie (2001) hizo una revisión de la literatura sobre el control químico de Hypsipyla spp., sumarizando que después de más de ocho décadas de la investigación en 23 países tropicales todavía no existía ningún método de control químico confiable, económico, y ambientalmente sano para prevenir daño económico por estos insectos. Él sugirió, sin embargo, que el control químico de estas plagas pudiera ser aplicable a las situaciones de viveros. Esto puede ser el caso en la Florida, en donde el taladrador de las meliáceas ataca las ramitas sobre todo en la primavera, y las aplicaciones químicas se pueden concentrar así durante este período.

Como la primer instar de la larva puede alimentarse en los tejidos superficiales por un breve período antes de taladrar en la ramita o la fruta (Ramírez Sánchez 1964), es teóricamente posible reducir a poblaciones del taladrador de las meliáceas por aplicaciones tópicos de un plaguicida o de un antifeedant'. En hecho, unas aplicaciones tópicas repetidas del azadirachtin (extracto de la semilla del neem ), un antifeedant' de insectos, a los árboles de caoba jóvenes durante el período principal del ataque de caoba del taladrador en la Florida (Abril-Mayo) redujeron la incidencia del daño (Howard 1995), pero un experimento similar después no fue conclusivo (Howard, inéditos). Algunos insecticidas del contacto que son eficaces contra otros taladradores de las ramitas han sido probados en el campo para reducir daño por el taladrador de meliáceas sin éxito. Un lavado de raíz con el imidacloprid antes de la estación de primavera no protegió las caobas contra los taladradores (Howard, inéditos). Sin embargo, puesto que el imidacloprid es absorbido en diferentes tasas por diversas especies del árbol, la eficacia de un lavado aplicado más anterior en la estación debe ser ensayado.

Control biológico. Cerca de 40 especies de insectos se han identificado como enemigos naturales del taladrador se las meliáceas en las Américas (Sands and Murphy 2001). Estas son, como el taladrador de las meliáceas sí mismo, nativas a la región. Son indudablemente de diferentes grados de importancia en regulación de poblaciones de este insecto, pero su efecto no es suficiente para prevenir daño económico. Aunque ha habido un cierto interés en las técnicas tales como el aumento de poblaciones de enemigas naturales, el control biológico del taladrador de las meliáceas no se parece una opción prometedora (vea revisión por Sands and Murphy (2001). Esta situación existe sobre todo porque incluso las poblaciones escasas pueden causar daño económico severo, según lo explicado previamente.

Silvicultura. Las caobas que crecen en bosques naturales ocurren a menudo en las bajas densidades y mezclados con muchas otras especies. Bajo tales condiciones son menos probables ser atacadas por los taladradores de las meliáceas. Este principio se ha aplicado en varias técnicas silviculturales con grados que variaban de éxito, y la investigación todavía sigue en esta área (Lamb 1966, Mayhew and Newton 1998, Hauxwell et al. 2001, Grogan et al. 2002). Se ha observado que en general las caobas que crecen en sombra tienden de ser menos susceptibles o escapan ataque de Hypsipyla spp., y las investigaciones recientes sobre el $H$. robusta indican que esta tendencia se debe a una diferencia fisiológica entre los árboles que crecen en sombra o en el abierto (Mahroof et al. 2000).

Mejoramiento de árboles. En la prueba de diversas procedencias de caoba y de cedros, menos daños se ha observado en algunas selecciones. La investigación para identificar razas genéticas de estos árboles que sean resistentes o que puedan superar ataque del taladrador de las meliáceas ha progresado bien (Mayhew and Newton 1998, Watt et al. 2001).

Manejo integrado. Las conclusiones de los participantes de un taller internacional sobre los taladradores Hypsipyla en 1996 eran que las estrategias más prometedoras eran relacionadas a la identificación y uso de genotipos resistentes, y el plantar de árboles de la caoba y del cedro en plantíos mezclados en cambio de plantíos puros y debajo de una canopia establecida. También fue acentuado que se debe promover el crecimiento vigoroso de árboles jóvenes por métodos culturales en el vivero y en plantaciones jóvenes. El control químico fue considerado como una herramienta para temporalmente reducir las 
poblaciones de los taladradores en áreas limitadas (Floyd 2001, Floyd and Hauxwell 2001, Speight 2001). Estas conclusiones se aplican sobre todo al manejo de las caobas y de otros árboles meliáceos cultivados para la madera. Manejo integrado en los viveros en la Florida en donde la caoba se cultiva para árboles de sombra debe involucrar con métodos de reducir a poblaciones de la plaga durante la primavera y con métodos de poda que promueven la recuperación de la forma de árboles atacados.

\section{Agradecimientos}

Estamos agradecidos a la Dra. Carrie Hauxwell, Senior Scientist, Agency for Food and Fibre Sciences, Department of Primary Industries and Fisheries, Brisbane, Australia, por su revisión y sus comentos sobre el manuscrito, y a la Dra. Marianne Horak, Especialista Taxonómica en Lepidoptera, Australian National Insect Collection, CSIRO Entomology Canberra, Australia, por información sobre el estado taxonómico actual de Hypsipyla spp.

\section{Referencias seleccionadas}

\section{Bauer GP. 1987. Swietenia macrophylla and} Swietenia macrophylla X S.mahagoni development and growth: the nursery phase and the establishment phase in line planting in the Caribbean National Forest, Puerto Rico. M.S. Thesis. State University of New York, College of Environmental Science and Forestry, Syracuse, N.Y.

Becker VO. 1976. Microlepidopteros asociados con Carapa, Cedrela, y Swietenia en Costa Rica, pp. 75-101. In Whitemore JL [ed.], Studies on the shootborer, Hypsipyla grandella (Zeller), Lep.:Pyralidae, CATIE Misc. Publ. No. 1. CATIE, Turrialba, Costa Rica.

Cintron BB. 1990. Cedrela odorata L. Cedro hembra, Spanish cedar, pp. 250-257. In Burns RMH, Barbara H [ed.], Silvics of North America 2: Hardwoods. Agricultural Handbook 644. United States Department of Agriculture, Washington, DC.

Entwistle PF. 1967. The current situation on shoot, fruit and collar borers of the Meliaceae., Proceedings of the 9th British Commonwealth Forestry Conference. Commonwealth Forestry Institute, Oxford.
Floyd RB. 2001. General conclusions and research priorities. pp. 183-187. In Floyd RB, Hauxwell C (eds.), International Workshop on Hypsipyla shoot borers in Meliaceae, 20-23 August 1996. ACIAR Proceedings No. 97.

Floyd RB., Hauxwell C. [eds.] 2001. Hypsipyla shoot borers in Meliaceaae. Proceedings of an International Conference held at Kandy, Sri Lanka 20-23 August 1996. ACIAR Proceedings No. 97. Australian Centre for International Agricultural Research, Canberra, Australia.

Griffiths MW. 2001. The biology and ecology of Hypsipyla shoot borers. pp. 74-80. In Floyd RB, Hauxwell C (eds.), International Workshop on Hypsipyla shoot borers in Meliaceae, 20-23 August 1996. ACIAR Proceedings No. 97.

Grijpma P. 1970. Immunity of Toona ciliata M. Roem. var. australis (F. v. M.) DC. and Khaya ivorensis A. Chev. to attacks of Hypsipyla grandella Zeller in Turrialba. Turrialba 20: 85-93.

Grijpma P. 1974. Contributions to an integrated control programme of Hypsipyla grandella (Zeller) in Costa Rica. Landbouwhogeschool te Wageningen., Wageningen, Netherlands.

Grogan J, Barreto P, Veríssimo A. 2002. Mahogany in the Brazilian Amazon: Ecology and Perspectives on Management. Imazon, Belém, Pará, Brazil.

Hauxwell C, Mayhew JE, Newton AC. 2001. Silvicultural management of Hypsipyla spp. pp. 151-163. In Floyd RB, Hauxwell C, (eds.), International Workshop on Hypsipyla shoot borers in Meliaceae, 20-23 August 1996. ACIAR Proceedings No. 97.

Holsten EH. 1976. Life cycle of Hypsipyla grandella (Zeller). pp. 112-116. In Whitemore JL [ed.], Studies of the shootborer, Hypsipyla grandella (Zeller) Lep. Pyralidae, CATIE Misc. Publ. No. 1. CATIE, Turrialba, Costa Rica.

Horak M. 2001. Currrent status of the taxonomy of Hypsipyla Ragonot (Pyralidae: Phycitinae). pp. 151-163. In Floyd RB, Hauxwell C (eds.), 
International Workshop on Hypsipyla shoot borers in Meliaceae, 20-23 August 1996. ACIAR Proceedings No. 97.

Howard FW. 1991. Seasonal incidence of shoot infestation by mahogany shoot borer in Florida. Florida Entomologist 74: 150-151.

Howard FW. 1995. Reduction in damage to mahogany shoot borer and mahogany leaf miner by use of azadirachtin. Journal of Tropical Forest Science 7: 454-461.

Howard FW, Solis MA. 1989. The distribution, life history, and host plant relationships of mahogany webworm. Florida Entomologist 72: 469-477.

Howard FW, Meerow AW. 1993. Effect of mahogany shoot borer on growth of West Indies mahogany in Florida. Journal of Tropical Forest Science 6: 201-203.

Howard FW, Gilblin-Davis R. 1997. The seasonal abundance and feeding damage of Hypsipyla grandella (Lepidoptera: Pyralidae) in seed capsules of Swietenia mahagoni in Florida. Florida Entomologist 80: 35-41.

Lamb FB. 1966. Mahogany of Tropical America. Its Ecology and Management. University of Michigan Press, Ann Arbor.

Mahroof RM, Hauxwell C, Edirisinghe JP, Watt AD, Newton AC. 2000. Effects of artificial shade on attack by the mahogany shoot borer, Hypsipyla robusta (Moore). Agricultural and Forest Entomology 4: 283.

Mayhew JE, Newton AC. 1998. The Silviculture of Mahogany. CABI Publications, Wallingford, UK.

Monte O. 1933. Hypsipyla grandella Zeller, uma praga da silvicultura (Lep. Phycitidae). Revista de Entomología (Río de Janairo) 3: 281-285.

Newton AC, Baker P, Ramnarine S, Mesén JF, Leakey RRB. 1993. The mahogany shoot borer: prospects for control. Forest Ecology and Management 57: 301-328.
Pennington TD. 1981. A monograph of the neotropical Meliaceae. New York Botanical Gardens, New York.

Ramirez Sanchez J. 1964. Investigación preliminar sobre la biología, ecología y control de Hypsipyla grandella Zeller. Boletín del Instituto Forestal Latino-Americano, Mèrida, Venezuela 16: 54-77.

Record SJ, Hess RW. 1943. Timbers of the New World. Yale University Press, New Haven, Connecticut.

Roovers M. 1971. Observaciones sobre el ciclo de vida de Hypsipyla grandella (Zeller) en Barinitas, Venezuela. Boletín del Instituto Forestal Latino-Americano de Investigación y Capacitación 38: $1-46$.

Sands DPA, Murphy ST. 2001. Prospects for biological control of Hypsipyla spp. with insect agents. pp. 121-130. In Floyd RB, Hauxwell C (eds.), International Workshop on Hypsipyla shoot borers in Meliaceae, 20-23 August 1996. ACIAR Proceedings No. 97.

Solomon JD. 1995. Guide to insect borers of North American broadleaf trees and shrubs. Agriculture Handbook 706. US Department of Agriculture, Forest Service, Washington, DC. Speight, M. R. 2001. Discussion summary: Integrated pest management of Hypsipyla spp. p. 179. In Floyd RB, Hauxwell C (eds.), International Workshop on Hypsipyla shoot borers in Meliaceae, 20-23 August 1996. ACIAR Proceedings No. 97.

Taveras R, Hilje L, Carballo M. 2004a. Development of Hypsipyla grandella (Zeller) (Lepidoptera: Pyralidae) in response to constant temperatures. Neotropical-Entomology 33: 1-6.

Taveras R, Hilje L, Hanson P, Mexzon R. 2004b. Population trends and damage patterns of Hypsipyla grandella (Lepidoptera: Pyralidae) in a mahogany stand, in Turrialba, Costa Rica. Agricultural and Forest Entomology 6: 89-98.

Tillmanns HJ. 1964. Apuntes bibliográficos sobre Hypsipyla grandella Zeller. Boletin del 
Instituto Forestal Latino-Americano, Merida,

Venezuela 14: 82-92.

Watt AD, Newton AC, Cornelius JP. 2001.

Research in resistance of mahoganies to Hypsipyla species. pp. 89-96. In Floyd RB, Hauxwell C (eds.), International Workshop on Hypsipyla shoot borers in Meliaceae, 20-23 August 1996. ACIAR Proceedings No. 97.

Wylie FR. 2001. Control of Hypsipyla spp. shoot borers with chemical pesticides: a review. pp. 109-115. In Floyd. RB, Hauxwell C (eds.), International Workshop on Hypsipyla shoot borers in Meliaceae, 20-23 August 1996. ACIAR Proceedings No. 97: 109-115. 